\title{
Miranda
}

Revue pluridisciplinaire du monde anglophone /

Multidisciplinary peer-reviewed journal on the English-

speaking world

18 | 2019

Guerre en poésie, poésie en guerre

\section{Notre Top 10 des films anglophones de 2018}

David Roche et Vincent Souladié

\section{QpenEdition}

\section{Journals}

Édition électronique

URL : http://journals.openedition.org/miranda/19089

DOI : 10.4000/miranda.19089

ISSN : 2108-6559

Éditeur

Université Toulouse - Jean Jaurès

Référence électronique

David Roche et Vincent Souladié, « Notre Top 10 des films anglophones de 2018 », Miranda [En ligne], 18 | 2019, mis en ligne le 17 avril 2019, consulté le 16 février 2021. URL : http://

journals.openedition.org/miranda/19089; DOI : https://doi.org/10.4000/miranda.19089

Ce document a été généré automatiquement le 16 février 2021.

\section{c) (i)}

Miranda is licensed under a Creative Commons Attribution-NonCommercial-NoDerivatives 4.0 International License. 


\title{
Notre Top 10 des films anglophones de 2018
}

\author{
David Roche et Vincent Souladié
}

En dépit des discussions sur l'avenir du cinéma, sur la place de Netflix et des autres plateformes, sur la mutation des modes de consommation et sur ce qui constitue un film à l'ère du numérique et d'Internet et du soi-disant post-cinéma, 2018 aura décidément été une année de haute tenue en termes de qualité de la production internationale, avec des films splendides comme Beoning (Corée du sud, Chang-dong Lee), Cold War (Pologne/UK/France, Pawel Pawlikowski), Leto (Russie/France, Kirill Serebrennikov) et Roma (Mexique/USA, Alfonso Cuarón, 2018). Le cinéma américain, australien et britannique n'aura pas été de reste non plus. Si des blockbusters comme The Meg (China/USA, Jon Turteltaub) n'ont fait que confirmer ce que l'on savait déjà que la bonhommie de Jason Statham ne suffirait jamais à nous faire avaler un énième requin en images de synthèse quelle qu'en soit la taille -, le succès de Black Panther (USA, Ryan Coogler) lui a permis d'être le premier film de superhéros nommé aux Oscars dans la catégorie Meilleur Film, et a posé des questions tout à fait pertinentes sur ce qui fait qu'un film est progressiste ou non, le blockbuster postulant implicitement qu'à l'image de Luke Cage, un superhéros noir n'est pas un superhéros universel. Green Book (USA, Peter Farrelly), qui a reçu l'Oscar du Meilleur Film, a aussi posé des questions intéressantes: tétanisée par le spectre de l'Oscar de Driving Miss Daisy (USA, Bruce Beresford, 1989), dont le feel-good movie de Farrelly renverse le postulat, la critique n'a pas toujours été sensible à la manière dont le film intègre la rencontre entre les personnages de Mahershala Ali et de Viggo Mortensen (tous deux excellents) dans des enjeux intersectionnels (classe sociale, ethnie, sexualité) qui complexifient le binarisme blanc/noir. À l'inverse, c'est la qualité artistique qui, aux yeux de nombreux critiques et internautes, aura été ignorée par l'Académie, concernant par exemple la prestation d'Ethan Hawke dans First Reformed (USA/UK/ Australie, Paul Schrader), récit d'un prêtre alcoolique en pleine crise de foi/e. Mary Queen of Scots (UK/USA, Josie Rourke) souffre d'une bande musicale lourde, et on peut se poser la question de la pertinence d'un tel film seulement cinq ans après le longmétrage du même nom de Thomas Imbach (Suisse/France); néanmoins, Saoirse Ronan 
et Margot Robbie assoient leur place parmi les actrices les plus talentueuses de leur génération. C'est aussi le talent des acteurs et actrices (KiKi Layne, Stephan James, Regina King, Colman Domingo - tous excellents) qui fait l'intérêt principal de If Beale Street Could Talk (USA), adapté du roman de James Baldwin, le très attendu successeur de Moonlight (USA, 2016), prouvant que, si le film a moins su séduire la critique, peutêtre en partie à cause de sa timidité structurelle, il prouve la capacité du metteur en scène Barry Jenkins à diriger des acteurs. L'adaptation du roman de Richard Ford, Wildlife (USA, Paul Dano), a elle aussi permis à Carey Mulligan et à Jake Gyllenhaal de briller à nouveau, malgré un film qui joue peut-être un peu trop la carte de la sobriété. Touchante découverte que celle de l'actrice Elsie Fisher et du réalisateur Bo Burnham, avec ce premier long métrage, Eighth Grade (USA), coming of age film qui, sur un mode plus naturaliste que Unfriended (USA, Levan Gabriadze, 2014) et moins brutal que Catfish (USA, Henry Joost et Ariel Schulman, 2010), pèse les potentialités d'expression et d'oppression des nouvelles technologies qui exacerbent les difficultés de la vie adolescente. Quant à l'acteur John Krasinski, il signe son troisième long métrage, $A$ Quiet Place (USA), un film d'horreur qui, comme son nom l'indique et comme le récent Don't Breathe (USA, Fede Alvarez, 2016), exploite le pouvoir du silence au cinéma. Certains cinéastes plus confirmés ont proposé des films intrigants. Avec The House That Jack Built (Danemark, France/Suède/Allemagne/Belgique), Lars von Trier reprend l'idée de la figure du tueur en série comme artiste - idée récemment exploitée par la série Hannibal (NBC, 2013-2015) - afin de proposer une métafiction qui explore la pulsion créatrice et destructrice. Steve McQueen, épaulé par Gillian Flynn, l'auteure de Gone Girl qui scénarise ici le roman de Lynda La Plante, propose avec Widows / Les Veuves (UK/ USA) (Viola Davis sublime) un thriller divertissant qui montre que la féminisation d'un genre n'est pas un simple renversement des genres sexuels mais exige une prise en compte de la diversité des expériences féminines, comme le pluriel du titre le suggère. Seul le temps pourra nous dire si Under the Silver Lake (USA) est bien le digne successeur du magistral It Follows (USA, 2015), mais il est au crédit de David Robert Mitchell d'avoir prolongé ses préoccupations majeures (la jeunesse et la culture populaire américaines contemporaines) tout en essayant d'explorer une veine du fantastique plus littéraire (influence de William Burroughs, Philip K. Dick ou encore Thomas Pynchon), comme avait pu le faire P.T. Anderson avec son adaptation d'Inherent Vice (USA, 2014). "Après un remarquable premier long métrage, Ex Machina (UK, 2014), Alex Garland renouvelle un schéma narratif que l'on croyait épuisé en plongeant un groupe de femmes scientifiques dans un environnement diurne et champêtre, baigné d'une lumière douce et d'une farandole de couleurs abstraites et changeantes suscitant un malaise diffus; Annihilation (UK/USA) offre ainsi une démarcation éco-fictionnelle efficace du modèle de la science-fiction horrifique proposé par Alien (Ridley Scott, 1979) et The Thing (John Carpenter, 1982), couplé aux questionnements philosophiques de Solaris (Andreï Tarkovski, 1972 ; Steven Soderbergh, 2002), et se donne à voir comme une œuvre dont l'iconographie inattendue et le ton dépressif et fataliste contrebalancent l'effet de déjà-vu qui ne nous quitte jamais vraiment». Enfin, Debra Garnik signe Leave no Trace (USA/Canada), film poignant sur la relation entre une adolescente et son père qui souffre de PTSD, et dont la dramaturgie centrée sur la tension entre amour et emprisonnement parvient à rester de bout en bout contenue dans un cadre naturaliste et à échapper au mélodrame, notamment grâce à un duo d'acteurs (confirmation pour Ben Foster, découverte de la jeune Thomasin McKenzie) époustouflant. 


\section{BlacKkKlansman / J'ai infiltré le Ku Klux Klan (USA, Spike Lee)}

2 Les longs métrages tournés par Spike Lee ces dix dernières années n'avaient pas vraiment attiré l'attention, si bien que son retour en grâce avec BlacKkKlansman n'a pas manqué de surprendre. Preuve qu'il est toujours maladroit de catégoriser une œuvre en cours, les derniers films de Lee à avoir connu un succès public et critique avaient été trop rapidement reçus comme des « œuvres de la maturité " $\left(25^{\text {th }}\right.$ Hour, 2002; Inside Man, 2006) en raison de leur ton sérieux et de la rigueur plutôt classique de leur mise en scène. Plus d'une décennie plus tard, BlacKkKlansman change de direction et retrouve la fraîcheur, la spontanéité, la modestie, l'humour juvénile, et aussi la hargne politique des premiers films. L'entreprise est pourtant sérieuse, sous la forme d'un buddy movie (porté par les excellents Adam Driver et John David Washington), il s'agit d'un polar d'infiltration dans les milieux du Ku Klux Klan à la fin des années soixante-dix, dont l'intrigue se conclue avec un intense suspense sur une tentative d'attentat. Mais Spike Lee ne se laisse pas enfermer dans un genre et dans une seule approche dramatique, il pratique un brassage de tonalités qui parviennent toutes à s'équilibrer : les codes de la blacksploitation y sont repris avec autant d'ironie que de nostalgie, le discours pamphlétaire y est asséné avec espièglerie, les congrégations racistes de l'Amérique profonde sont tournées en ridicule sans perdre de leur pouvoir d'inquiétude et d'horreur. La légèreté insuffle alors un rythme, agrémenté d'une riche bande musicale, grâce auquel les idées politiques du cinéaste sont assenées sans solennité mais avec urgence et sincérité. Les historiens du cinéma pourront trouver à redire à la vision a priori caricaturale que Spike Lee donne du cinéma classique hollywoodien et de la vision raciste que celui-ci présentait de l'esclavage. BlacKkKlansman s'ouvre ainsi par une séquence d'Autant en emporte le vent (Victor Fleming, 1939), tandis que Naissance d'une Nation (D.W. Griffith, 1915) est regardé et célébré religieusement par les personnages pro-aryens du film. Même s'ils étaient depuis longtemps tombés de leur piédestal classique, ces deux modèles sont frontalement attaqués et présentés aux jeunes générations de spectateurs comme l'exemple d'un cinéma qu'il faut dénoncer, sinon oublier pour avancer. Pourtant, le propos n'est peut-être pas si transparent. La modernité exaltante de la mise en scène de Spike Lee ne l'empêche pas non plus d'avoir recours à des moyens formels qui ont fait leur preuve depuis bien longtemps. Ainsi la montée en puissance du suspense lors de la séquence du climax final se fonde sur un rigoureux montage alterné, dont on se souvient qu'il avait trouvé son premier usage stimulant dans la dernière partie de Naissance d'une Nation. Spike Lee ne peut plus décréter innocemment que tous les cinéastes doivent quelque chose à Griffith, comme Orson Welles ou Stanley Kubrick l'avaient déclaré avant lui, puisqu'il attaque très ouvertement le contenu on ne peut plus controversé de ce film séminal, mettant en scène la naissance épique et salvatrice du Ku Klux Klan. En revanche, l'invention esthétique de ce film s'expose comme un legs immuable dont il convient d'utiliser le pouvoir d'exaltation idéologique à de nouvelles fins. Le montage alterné comme prise de guerre en somme. Plus pugnace et impétueux que jamais, Spike Lee tombe en revanche dans des travers prosélytes qui n'étaient pas du tout nécessaires lorsqu'il choisit de conclure son film par des images d'archives des émeutes racistes survenues à Charlottesville en 2017. Ce point d'orgue, déjà présent dans le livre originel de Ron 
Stallworth, apparaît redondant et exige du public qu'il quitte la salle avec un sentiment d'indignation univoque et pompeux, que la mise en scène était déjà parvenue à inspirer de manière plus subtile.

\section{The Favourite / La Favorite (Irlande/UK/USA, Yorgos Lanthimos)}

3 À ce stade de sa carrière, Yórgos Lánthimos ne surprend plus autant qu'il avait pu le faire avec Canine (2009) ou The Lobster (2015). C'est bien le moindre reproche que l'on peut faire à son dernier opus, dont il n'a pourtant pas signé le scénario (écrit par Deborah Davis et Tony McNamara): le caractère sulfureux qui vient lentement bousculer les codes canoniques du film en costume feutré est attendu dès le départ. Cela n'empêche pas The Favorite de proposer une reconstitution jubilatoire de la royauté décadente de la Reine Anne et de ses relations de dépendance amoureuse avec ses sujettes, dans la Grande-Bretagne du début du XVIIIème siècle. Bien sûr, Lánthimos n'a aucun intérêt à s'en tenir à la vérité historique et à la traiter avec respect et authenticité, il ramène plutôt celle-ci à sa manière personnelle de dépeindre le monde et puise dans l'anecdote et la légende de quoi nourrir son humour noir et ses obsessions artistiques, un peu comme avait pu le faire Sofia Coppola avec Marie Antoinette (2006), dans un registre très différent. L'autarcie du huis-clos ridiculise le pouvoir des décisions militaires et politiques, jeu désincarné auquel la reine, ogre puéril excellemment interprétée par l'actrice oscarisée Olivia Colman, participe à peine. Par ailleurs, Emma Stone et Rachel Weisz excellent dans leur rôle de favorites manipulatrices se disputant le rôle de la plus perverse. Les personnages masculins qui les entourent sont volontairement fades et inconsistants, ils n'ont pas même le bénéfice de la médiocrité ou du ridicule. Les actrices ont ainsi toutes la place pour développer la richesse de leur jeu et imposer leur présence physique, que Lánthimos n'érotise jamais, contrairement à ce que son sujet pourrait lui dicter, préférant donner à leurs corps une densité charnelle dérangeante. Pour donner vie à ce nouvel environnement claustrophobe régi par des règles saugrenues et malsaines, comme l'étaient ceux de ses films précédents, la mise en scène de Lánthimos n'est pas modeste mais aussi emphatique et expressive qu'elle pourrait l'être chez Kubrick, à qui il emprunte quelques idées formelles (les travellings virtuoses dans les couloirs du palais, l'usage d'objectifs déformants, le grand angle et la géométrisation du décor, les éclairages clair-obscurs). Cette emphase, aidée d'une bande-son oppressante, procure plus que jamais le malaise tant elle met en valeur l'outrance des situations, leur grotesque ou leur violence psychologique.

\section{First Man / First Man : le premier homme sur la lune (USA/Japan, Damien Chazelle)}

4 Grand oublié des Oscars 2019, ce biopic du jeune prodige Damien Chazelle sur l'astronaute Neil Armstrong se présente comme l'anti-Apollo 13 (USA, Ron Howard, 1995) : à l'instar de l'envoutant Jackie (USA/France/Chilie/Hong Kong/Allemagne/UK, Pablo Larraín, 2016), il s'agit d'interroger une figure mythique américaine, et de l'écarter du mythe en la révélant dans ses contradictions proprement humaines. 
Plastiquement, l'Armstrong interprété par Ryan Gosling a tout du héros hollywoodien, certes, mais le protagoniste est avant tout un chercheur qui se réfugie dans le travail et qui est mu par un idéal non pas patriotique mais scientifique : il n'adhère pas à l'image du cowboy kennedien affrontant l'ennemi soviétique, mais à une vision de l'effort collectif dans lequel les découvertes soviétiques sont un apport précieux. Comme dans certains films de guerre contemporain, dont The Hurt Locker / Démineurs (USA, Kathryn Bigelow, 2008), l'héroïsme s'avère relever de la pathologie sans qu'il y ait pour autant jugement moral sur la personne. Mais ce n'est pas tant ça qu'on retiendra de First Man. Le troisième film de Chazelle est une expérience kinétique, où l'on vit chaque expérience scientifique de l'intérieur. C'est un film qui s'efforce de transmettre le vertige du ciel et de l'espace à travers une esthétique sensorielle : caméra tremblante, gros plan claustrophobe qui contraste avec les plans généraux de l'immensité des cieux, et bien sûr, de la part de celui qui a été révélé avec les films musicaux Whiplash (USA, 2014) et $\mathrm{La}$ La Land (USA/Hong Kong, 2016), disjonctions auditives entre le vacarme et un silence tout aussi menaçant. First Man est l'objet parfait pour une appréhension du cinéma comme "expérience incarnée ", approche très prisée dans les études cinématographiques nord-américaines. Dès la scène d'ouverture, nous, spectateurs confortablement installés dans une vaste salle de cinéma, sommes transportés dans l'espace réduit et fragile du cockpit, alors que les bruits environnants de tôles nous font ressentir la fragilité de ce qui nous sépare du vide. Le jeu tout en contrôle de Ryan Gosling exprime, d'une part, l'entêtement du scientifique, mais au niveau sensible, ce visage en gros plan est aussi la surface, voire l'écran, sur laquelle nous projetons nos angoisses, nos interprétations et, in fine, nos idéaux et notre idéologie. L'esthétique kinétique et les sublimes scènes d'action font plus que structurer le film, elles en sont le fond même. En nous donnant accès à l'intériorité d'Armstrong et en insistant sur l'écart entre les discours politiques et scientifiques et l'expérience intime, la conclusion du film nous invite alors à reconsidérer notre rapport aux figures réelles et imaginaires qui éveillent nos désirs et que nous édifions en mythe, plus précisément à la manière dont nous vivons, ressentons à travers elle.

\section{Hereditary / Hérédité (USA, Ari Aster)}

5 La voie ouverte par le cinéma fantastique américain des années 2010 est absolument passionnante. Alors qu'on aurait pu le croire balisé de toute part, le genre parvient encore à surprendre par son exigence et son imprévisibilité. Quelles que soient leur différences, David Robert Mitchell, Robert Eggers, Alex Garland, Jordan Peele, David Lowery et Ari Aster (qui signe avec Hérédité son tout premier long métrage) seront bientôt identifiés comme une génération innovante qui a su se tenir à l'écart des modes commerciales, incarnées par James Wan et ses épigones, pour réinventer un fantastique intellectuel et expérimental. De par son phénomène générationnel, et sans fer de lance, ce renouveau est sans doute plus important encore que ne le fut la découverte des premiers films américains de M. Night Shyamalan il y a vingt ans, lequel incarnait un peu à lui seul une ambition artistique comparable à ce qui survient aujourd'hui (alors qu'il s'avère à la vision de Glass que Shyamalan n'est plus en phase avec la rigueur et l'inventivité actuelle). Concernant Hérédité, tout s'est joué pour nous dès les premières secondes du film, et sans même savoir quelle serait l'intrigue. Alors que le film s'ouvre en plan d'ensemble sur une grande maison de poupées, la caméra s'en approche lentement pour nous faire contempler la précision des détails décoratifs de son 
intérieur miniature. Nous nous sommes dit que si ce plan a priori banal se concluait de manière aussi intelligente et audacieuse que nous étions en train de l'anticiper, le film mériterait de figurer dans le top de l'année. N'en disons pas plus. Difficile d'ailleurs de décrire une œuvre qui propose tant de retournements de situation que l'on est loin de tous voir venir. La première partie, mélodrame familial anxiogène, fascine par sa lenteur et son retour aux sources du gothique, tandis que le virage surprenant qui survient à mi-film est de nature à dérouter irrémédiablement l'audience. Il est pourtant remarquable de voir un cinéaste pousser le spectateur hors de sa zone de confort et faire librement évoluer son récit filmique vers une exubérance qui pourrait paraître incongrue. Quoiqu'il en soit, tout ne repose pas sur ces surprises dramatiques mais sur la manière unique qu'a Ari Aster d'instiller une atmosphère suffocante à partir d'une raideur froide des mouvements et d'une exploitation inconfortable du silence. Toni Colette (évidemment empruntée au Sixième Sens de Shyamalan) apporte à son jeu une fébrilité que contrebalance la profondeur et la rationalité de Gabriel Byrne, mais c'est la jeune actrice Milly Shapiro qui vole la vedette aux acteurs confirmés. Son personnage de freaks ne ressemble en rien aux adolescents auxquels le fantastique domestique nous demande en général de nous identifier. L'enfance n'est plus un territoire innocent en proie aux cauchemars, elle fait littéralement corps avec la terreur.

\section{Isle of Dogs / Lîle aux chiens (Allemagne/USA, Wes Anderson)}

6 Il est possible que le neuvième long métrage de Wes Anderson soit un ton légèrement en-dessous de ses grands chefs d'œuvre que sont Moonrise Kingdom (USA, 2012) et The Grand Budapest Hotel (USA/Allemagne, 2014). Mais l'énergie du film n'en est pas moins puissamment jubilatoire. Il n'y a pas tromperie sur la marchandise : qui vient chercher du Anderson trouvera du Anderson. D'un point de vue esthétique, il s'agit avant tout d'une synthèse de Fantastic Mr. Fox (USA, 2009), première incursion dans l'animation «stop motion » du cinéaste américain, et de The Grand Budapest Hotel, dont il emprunte à la fois le rythme, les décors miniatures, le festival chromatique, et l'approche fabulatrice au contexte historique. Cette fois-ci, la poétique andersonnienne, avec ses plans frontaux et ses dialogues énoncés sur un ton laconique, est mise au service de la quête d'un garçon japonais nommé Atari pour son chien, Spots, qui l'amènera sur une île où les chiens ont été mis en exil suite à une épidémie de chien fou (« dog flu »); face à cette preuve que l'homme demeure le meilleur ami du chien malgré la politique discriminatoire du maire de Megasaki, une meute de chiens abandonnés finira par apporter son aide à l'enfant. Cette quête sur fond de dystopie propose ainsi une allégorie qui, grâce à la figure anthropomorphisée du chien, le meilleur ami de l'homme, parvient à fusionner les préoccupations contemporaines, d'une part pour le monde animal et par extension pour l'environnement, et d'autre part pour les catastrophes humanitaires comme le sort des réfugiés. L'île apocalyptique est donc à la fois un monde pollué et un camp de concentration. Et la politique du maire y est, sans surprise, fondée sur l'oubli, voire le déni d'un quelconque problème et de l'« humanité » des chiens. Une attitude que l'on retrouve chez un grand nombre des puissants de ce monde. Or, c'est ici que le défi technique et esthétique fait partie liée avec le propos : le projet est d'insuffler la vie non seulement dans l'animal mais dans sa version artificielle assumée. C'est à ce niveau-ci que le casting de choc joue un rôle 
central. Car les voix de Bryan Cranston (Chief), d'Edward Norton (Rex), de Jeff Goldblum (Duke), de Bill Murray (Boss), de Frances McDormand (Interpreter Nelson), ou de Scarlett Johansson (Nutmeg) sont pleinement identifiables, et les acteurs s'en donnent à cœur joie pour animer les visages souvent très figés des chiens avec des modulations de voix discrètes qui n'enfreignent pas le ton anti-mélodramatique andersonien. Enfin, cette chorale de voix de stars américaines permet de mieux mettre en valeur la voix du jeune acteur Koyu Rankin, et donc la voix de l'autre (l'humain, l'enfant, le japonais). C'est maintenant avec impatience que nous attendons de voir comment Anderson va explorer l'histoire de son pays d'adoption, la France, dans The French Dispatch, dont la sortie est prévue en 2020.

\section{The Mule / La Mule (USA, Clint Eastwood)}

7 Il est habituel de reconnaître que la part la plus passionnante de l'œuvre de Clint Eastwood réside dans l'autoportrait ambigu qu'il y dessine en filigrane depuis ses débuts. Or, d'Impitoyable à Gran Torino, voilà plusieurs décennies qu'il continue de se filmer dans des fictions crépusculaires qui semblent signer à chaque fois ses adieux à l'écran. Jamais cinéaste n'a autant sublimé sa sortie, tout nouvelle agonie étant pour lui l'occasion de se réinventer. Nul ne peut prédire si The Mule sera bel et bien son dernier rôle, mais il fait à nouveau de celui-ci l'incarnation de valeurs anciennes qu'il confronte à l'ordre nouveau du cinéma et de la société, pour poser une fois encore cette question coutumière : que reste-t-il aujourd'hui de Clint Eastwood? Ses apparitions en tête d'affiche d'un nouveau film annoncent toujours un rendez-vous avec les restes de son image, donnant lieu en guise de constat à une problématique figurative et dramatique. Tout le capital sympathie du personnage provient de cette nonappartenance résolue à un monde qui le dénigre et qu'il prend de haut tout en essayant de le rattraper à son rythme. De même qu'Eastwood manifestait dans The Rookie (1990) son essoufflement face aux buddy movies d'action des années 1980, qui avaient su damer le pion au cinéma dont il était autrefois le maître, on pourrait reconnaitre avec une certaine ironie que la lenteur et la mélancolie de The Mule en fait l'anti Fast \& Furious, sa réponse inattendue aux actioners motorisés qui prennent le narco-trafic à la frontière mexicaine comme cadre narratif exotique et spectaculaire. Le film n'en est pas moins dynamique, il donne même des leçons quant à la manière de réguler la tension dramatique par le montage sans gaspillage d'énergie, en maniant avec aplomb l'art de l'ellipse et de l'esquisse. Eastwood a compris depuis longtemps qu'il ne pouvait continuer à exister à l'écran qu'en tant que contre-modèle, face aux générations successives de héros d'action, auxquels il n'a plus de compte à rendre depuis longtemps, et face à sa propre image figée dans le passé des années 1970, à laquelle il se sait toujours comparé aujourd'hui (jusqu'à il y a 10 ans, un énième Dirty Harry était encore réclamé par les fans). Dans Gran Torino, Eastwood défiait encore ses adversaires de son regard d'aigle et de son index tendu. Dans The Mule, l'acteur force le trait du dépérissement physique (s'il exhibe sans gêne la peau vieillie de son corps à demi nu, cette démarche lente et voutée n'est pas la sienne) et se présente pour la première fois comme une figure douloureusement fragile. Le cinéaste s'amuse avec malice de l'ambiguïté insoluble de la double image humaniste et réactionnaire avec laquelle il a forgé sa persona, et que sont venus récemment brouiller ses prises de positions politiques en public, voire ses films dont les nuances étaient plus difficiles à cerner (American Sniper, 2014; The 15:17 to Paris, 2018). En redevenant une figure filmique en 
chair et en os dans The Mule, Eastwood sait très bien qu'il se rend à nouveau insaisissable et intouchable.

\section{The Rider (USA, Chloé Zhao)}

8 Brady Blackburn, un cavalier spécialisé dans le bronc riding (sans selle), voit sa carrière, sa passion et son identité remises en question par une chute qui lui provoque une fracture crânienne : finira-t-il par remonter à cheval au prix de sa vie ? C'est cette histoire simple que raconte le deuxième long métrage de Chloé Zhao, réalisatrice chinoise relocalisée dans le Dakota. Zhao a un talent rare pour rendre évidente des situations sensibles, pour faire jaillir la poésie de l'ordinaire, comme pour diriger des acteurs non professionnels dans leur milieu d'origine, à commencer par le touchant Brady Jandreau dans le rôle principal. Avec son premier film, Songs My Brothers Taught $\mathrm{Me}$ (2015), The Rider forme une sorte de diptyque sur la réserve Pine Ridge du Dakota du Sud habitée par les Lakota Sioux. On y décèle une persistance du souffle mythique de l'Ouest régénérée non pas par la violence, comme le voulait Theodore Roosevelt, mais par la rencontre entre les cultures, plus proche du modèle de la frontera proposée par la poétesse Gloria Anzaldúa (voir l'article d'Hervé Mayer dans ce numéro de 2019-04-17T17:19:00 Miranda). Les amérindiens que documente Zhao vivent dans des conditions économiques difficiles, mais démontrent également une expérience culturelle riche et hybride qui intègre les contraires: cultures cowboy et traditionnelles, heavy metal, hip-hop et chants traditionnels, intégration dans l'espace traditionnel et dans une société qui a transformé l'Ouest en spectacle. Ce qui pose problème, ce ne sont pas tant les tensions liées aux politiques identitaires que la vie elle-même, la survie, et donc les choix éthiques. Et c'est dans cette question de l'invention de soi, de l'agentivité, que les champs éthiques, politiques et poétiques se rejoignent. C'est que, pour le Rider, ces éléments ne sont pas en contradiction mais existent dans un plan d'immanence : le spectacle qu'offre le rodéo ne s'inscrit pas tant dans une compétition masculine entre cowboys (on n'est dans l'émulation du dépassement de soi plus que dans la compétition individuelle) que dans cette relation intime que Brady Blackburn et ses amis entretiennent avec le monde naturel, comme ce cheval caractériel qu'il apprivoise dans une séquence quasi-documentaire. Avec une caméra qui colle aux corps tout en les intégrant harmonieusement à leur environnement, Zhao dépeint un Ouest qui retient toute sa poésie, mais débarrassé du sensationnalisme du mythe et de son idéologie, pathétique et nostalgique, de la masculinité dure. C'est ainsi qu'à travers le choix effectué dans le film par le personnage de Blackburn, The Rider se démarque délibérément du splendide The Wrestler (Darren Aronofsky, 2008), comme l'a d'ailleurs souligné la cinéaste elle-même.

\section{The Sisters Brothers / Les Frères Sisters (France, Espagne, Roumanie, USA, Jacques Audiard)}

Quoi de mieux que de se lancer dans son premier long métrage en anglais sinon en se prêtant au plus américain des genres, le western? C'est le choix qu'a fait Jacques Audiard, en collaboration avec son scénariste attitré Thomas Bidegain, en portant à l'écran le roman du canadien Patrick DeWitt. Porté par un casting de rêve - John C. Reily, Joaquin Phoenix, Jake Gyllenhaal et le moins connu mais tout aussi excellent Riz 
Ahmed -, Les Sisters Brothers est peut-être le plus grand western depuis The Assassination of Jesse James by the Coward Robert Ford / L'Assassinat de Jesse James par le lâche Robert Ford (USA/Canada/UK, Andrew Dominik, 2007). La force du film vient tout d'abord d'un récit qui subvertit les conventions narratives et idéologiques du western à chaque point tournant tout en maintenant une logique implacable. Loin des thèses de Theodore Roosevelet sur la force régénératrice de la violence et de son application assez stricte dans le western classique ou de son détournement parfois nihiliste dans le western italien ou révisionniste, les frères Sisters, chasseurs de prime des plus convenus, vont, à la suite du détective John Morris, effectuer le parcours du renoncement de l'individualisme et de la violence - et donc de la chasse à l'homme - pour embrasser le travail collectif, parcours qui les amènera à revenir vers une sérénité féminine inscrite dans leur nom de famille même. La bifurcation narrative sur l'orpaillage est un véritable morceau de bravoure narratif, le cinéma classique ayant généralement employé la chasse à l'or comme un arrière-plan à un récit à la dramaturgie plus sensationnelle (The Hanging Tree / La colline des potences [USA, Delmer Daves, 1959]) ou comme une alternative possible à la trajectoire du héros (Bend of the River / Les affameurs (Antony Mann, 1952)] - l'une des meilleures histoires du sympathique film anthologique The Ballad of Bustes Scruggs / La ballade de Buster Scruggs (USA, Ethan et Joel Coen) s'empare également de ce sujet, avec un Tom Waits tout en grimaces et grognements. Le film offre également des moments de poésie inoubliables, à commencer par le gunfight nocturne inaugural dans lequel l'écran plongé dans un noir total est subitement illuminé par des jets de feu. Cette poésie culmine avec la première démonstration de la solution inventée par Hermann Kermint Warm pour révéler la présence de l'or dans une rivière, qui trace des nuées vertes à l'écran - scènes qui font basculer le récit dans le fantastique, la folie de l'or donnant lieu à une fantaisie scientifique digne d'Edgar Poe. Le tout est renforcé par une musique composée par Alexandre Desplat, fraichement oscarisé pour The Shape of Water (USA, Guillermo del Toro, 2017, tantôt entrainante, tantôt lugubre, où le mélodique est contaminé par la dissonance. On l'aura compris, avec ses qualités à la fois actorales, narratives et plastiques, le sans faute d'Audiard et de son équipe, récompensé par quatre fois aux Césars (dont meilleur réalisateur et meilleure photographie), est de toute évidence un autre grand oublié des Oscars 2019.

\section{Sorry to Bother You (USA, Boots Riley)}

10 Premier long métrage du rapper et militant afro-américain, Sorry to Bother You est une fable hilarante, branchée, politique, délirante, délicieuse, qui s'inscrit dans une longue tradition de la satire anglo-saxonne remontant au moins jusqu'à Jonathan Swift, l'auteur des Voyages de Gulliver (1726) et de la "proposition modeste » (1729) (pour résoudre le problème de la pauvreté en Irlande, il suffirait de dévorer leurs enfants). Le récit se déroule à Oakland, la ville des Blank Panthers. Cassius Green, un trentenaire à la peine dont le nom associe deux icônes afro-américains (Mohammed Ali et Al Green), accepte un emploi dans un service de télévente. Après des débuts laborieux, il finit par suivre le conseil d'un vieux sage du métier, Langston (Danny Glover), qui lui conseille de " prendre sa voix de blanc " pour améliorer son taux de ventes. Le succès de Cassius est alors quasi-immédiat et entre en conflit avec la lutte des employés de la boîte, menéé par un syndicaliste coréen-américain nommé Squeeze, et soutenue par la petite amie de Cassius, une artiste nommée Detroit (Tessa Thompson). Tous les ingrédients 
sont réunis pour un double récit classique articulant la romcom avec le film militant, où le protagoniste doit choisir entre personnel ou collectif. Ce choix est incarné par la figure de l'artiste féministe, dont le nom renvoie à une ville dont l'histoire est empreinte d'enjeux économiques (l'industrie automobile) et raciaux (voir le récent film de Kathryn Bigelow). Mais c'est alors que Boots Riley décide de faire appel à l'esprit de Swift pour dériver vers un troisième genre : la SF horrifique ainsi que le film de zombie classique (comme White Zombie [Victor Halperin, 1932]) qui associe le maitre des zombies à un capitaliste. L'hybridation générique du film (qui concerne aussi la bande son) est alors entièrement mise au service d'une vision politique à la fois post-marxiste et intersectionnelle, preuve s'il en faut que le post-modernisme n'est en rien synonyme de désengagement politique. C'est ainsi que Sorry to Bother You examine l'articulation entre les questions raciales, sociales et féministes et les incorpore, à travers les écarts générationnels entre certains personnages, dans une perspective historique spécifiquement américaine. Le film, avec ses références intertextuelles et notamment la référence à Swift, s'inscrit à son tour dans une dénonciation de l'histoire mondiale de l'oppression d'un groupe privilégié sur la majorité des humains dont les pratiques néolibérales gloablisées contemporaines ne sont qu'une nouvelle déclinaison. Parce que le pouvoir qu'on fait miroiter à Cassius ne relève aucunement de l'agentivité : bien au contraire, il est synonyme d'assujettissement total et d'exploitation, comme quand on l'oblige à rapper simplement parce qu'il est noir. C'est ainsi que Sorry to Bother You développe, à notre sens, un propos d'une subtilité bien plus grande que le plus convenu mais néanmoins touchant The Hate U Give (USA, George Tillman Jr.). Boots Riley nous met alors face à un paradoxe concernant l'art politique, soulevé par les films de Quentin Tarantino : que la nuance que requiert une perspective intersectionnelle passe peut-être par le grotesque, le monstrueux, l'excès, le fantastique et l'hybridité, autrement dit par des modes, des figures ou des esthétiques qui mettent justement en tension les limites de l'identité.

\section{Sweet Country (Australie, Warwick Thornton)}

11 Retour magistral du chef-op/réalisateur aborigène, huit ans après le magnifique Samson $\&$ Delilah (2009). Primé à plusieurs festivals australiens et vainqueur de la caméra d'Or à Cannes, ce troisième long métrage a malheureusement connu une brève vie en salle et a été rapidement distribué sur Netflix, même s'il demeure plus visible que le très réussi western sud-africain Five Fingers for Marseille (Afrique du Sud, Michael Matthews, 2018), avec son allégorie qui semble confirmer les thèses de Benedict Anderson pour qui une nation naît d'une lutte fraternelle. Avec Sweet Country, Thornton nous offre un western du point de vue aborigène et s'inscrit en ce sens dans la filiation du Tracker (Australie, 2002) de Rolf de Heer. La déconstruction du western passe par un renversement du postulat de The Searchers / La Prisonière du désert (1956) de John Ford: ici, c'est un indigène, Sam Kelly, qui a commis un meurtre par légitime défense qui est pourchassé par un homme de loi fou. Mais l'hommage vise avant tout à souligner la différence : l'homme blanc australien ne sera jamais l'américain mythique qui connaît les indiens, il ne parviendra jamais à maîtriser le territoire australien et ne devra sa vie qu'à la clémence de Sam Kelly. En nommant son héros après le célèbre hors-la-loi australien qui, en 1906, était justement le sujet du premier film australien - lequel est d'ailleurs projeté dans le village -, Thornton inscrit son récit dans une histoire cinématographique résolument australienne et postcoloniale, notamment avec cette 
scène qui inverse celle de Lawrence d'Arabie (David Lean, 1962) quand l'indigène sauve l'homme blanc dans un désert de sable blanc sous un soleil oppressant. Mais c'est avant tout son propre projet esthétique et politique que poursuit Thornton avec Sweet Country, à travers un remarquable travail plastique, aussi bien visuel que sonore. Samson \& Delilah avait déjà démontré la sensibilité musicale du chef opérateur et son sens du rythme. Sweet Country prolonge le travaille sur la rupture, notamment avec ces images (flashbacks? flashforwards? fantasmes ?) qui viennent briser la marche linéaire du récit colonial et introduire une sensibilité temporelle plus proprement aborigène, puisque l'incertitude demeure quant au statut de certaines images. Thornton introduit ainsi la résistance au récit dominant au niveau diégétique, à travers son héros, mais surtout au niveau de la narration, à travers le travail plastique. Thornton compte parmi des cinéastes aborigènes de talent, dont Wayne Blair et Ivan Sen, mais son pays tient peut-être avec lui son plus grand réalisateur depuis Peter Weir, et voit ainsi sa politique volontariste de valorisation de la culture aborigène récompensée.

\section{INDEX}

Mots-clés : cinéma américain, cinéma australien, cinéma britannique, BlacKkKlansman : J’ai infiltré le Ku Klux Klan, La Favorite, First Man : le premier homme sur la lune, Hérédité, L'île aux chiens, La Mule, The Rider, Les Frères Sisters, Sorry to Bother You, Sweet Country

Keywords : American cinema, Australian cinema, British cinema, BlacKkKlansman, The Favourite, First Man, Hereditary, Isle of Dogs, The Mule, The Rider, The Sisters Brothers, Sorry to Bother You, Sweet Country

Thèmes : Film

\section{AUTEURS}

\section{DAVID ROCHE}

Professeur

Université Toulouse- Jean Jaurès

mudrockca@gmail.com

\section{VINCENT SOULADIÉ}

Maître de Conférence

Université Toulouse- Jean Jaurès

souladie.vincent@gmail.com 